\title{
Slope anchor cable life evolution model and prediction ${ }^{1}$
}

\author{
Jing Wang ${ }^{1,}$, Jiwei Liu ${ }^{1, b}$ and Bing Liu ${ }^{1, c}$ \\ ${ }^{1}$ School of Automation \& Electrical Engineering, University of Science and Technology Beijing, \\ Beijing 100083, China; \\ austb_wangjing@126.com, beasyliu@vip.sina.com, custb_lb_1001@163.com
}

Keywords: Slope anchor, life prediction, reliability theory, SVM

\begin{abstract}
After a comprehensive analysis of the characteristics of slope anchor structure, a mature reliability theory model in durable life prediction field is applied to predict the life of this structure, and support vector machine method is used to solve the probability density estimation for predicting factors in the prediction model in case of a small sample; therefore, the availability of reliability theory life prediction method is improved in case of a small sample. Finally, reliability theory and K-S test method are used to predict and test the life based on the sample data from Qinshan Nuclear Power Plant.
\end{abstract}

\section{Introduction}

Geotechnical anchorage technology is widely applied in China, whose advancement, scientificity and simplicity are beyond doubt. However, as a covert project, the distinctive characteristic of anchor structure is hidden, and there are strict requirements for durability in the inclement environment.Since the lack of knowledge of durability and inadequate attention in the past, as time goes by, the problem with durability of anchor structure has become increasingly evident. To determine correctly the normal functions of anchor structure, i.e., if safety, serviceability and durability meet the design, construction, use and other requirements can expose potential hazards and prevent a major accident, and the research results can be directly used for structural design; it has a very important practical significance in improvement of design standards and construction quality of a project. The greatest threat to the life of anchor structure is anchor rod corrosion, and therefore, the study on the life of anchor structure focuses on its corrosion.

Professor Mehta pointed out in the keynote report titled Durability of concrete - Fifty years of progress ${ }^{[1]}$ that: "The causes of damage to concrete in the world are in descending order of importance: Reinforcement corrosion, frost damage, and physical and chemical effects." Among the factors causing reinforcement corrosion, the role of chloride ion corrosion is the most significant; since degradation of durability of concrete structure due to chloride ion corrosion has always been a very serious problem in the engineering construction field, which greatly harms the reliability and safety of the structure.

This paper summarizes the research results of concrete corrosion in the Materials Science, takes advantage of the characteristics of geotechnical anchor structure to determine chloride ion corrosion as the main cause affecting anchor life and develop a geotechnical anchor structure life evaluation method thereon.

\section{Prestressed anchor rod corrosion mechanism}

\subsection{Construction of anchor structure}

Geotechnical anchor structure ${ }^{[2]}$ is to interlock tightly a structure and a formation by use of anchor bar embedded in the formation and use the tension of friction transfer structure between anchor bar and surrounding formation to maintain the stability of structure and rock and earth mass. Anchor bar

\footnotetext{
${ }^{1}$ This paper supported by the National Program on Key Basic Research Project (973 Program) (Grant No.
} 2011CB013502-3) 
is inserted into a pre-drilled hole, whose bottom end is fixed; after fixing, it is usually pre-stressed. The end of anchor bar exposed on the ground is fixed with anchor head.

\subsection{Anchor structure corrosion}

The anchor structure is mainly composed of metal and grouting. Anchor rod is tightly wrapped by grouting, whose corrosion therefore mainly occurs during the contact with the grouting; cement mortar is the most common grouting ${ }^{[3]}$; therefore, reference may be made to the reinforced concrete research results for analysis of anchor cable corrosion in the environment where it is wrapped by the grouting $^{[4]}$.

(1) Reinforcement corrosion due to chloride ion corrosion

Chloride is an depassivator. After the invasion of chloride ions to the surface of reinforced concrete, they are attached to the passivation film and cause the $\mathrm{PH}$ value at this position to drop rapidly, damaging the passivation film on the reinforcement surface and leading to reinforcement corrosion ${ }^{[5]}$. It is shown that corrosion by chloride ion is the major factor of reinforcement corrosion $^{[6]}$, and the life of reinforced concrete can be reliably predicted through study of chlorine ion.

(2) Transfer of chloride ions in concrete

Chlorine ions are internally transferred from the ambient environment through internal voids and microcrack system in the concrete to the concrete. In most cases, the diffusion effect is considered to be the predominant mode of transfer.

(3) Chloride ion diffusion theory model

For chloride ion diffusion of concrete, the diffusion model based on Fick's second law is the most commonly used in the studies, which will also be used in this paper. Expression of Fick's second law [7] is:

Its solution is:

$$
\frac{\partial c}{\partial t}=\frac{\partial}{\partial x}\left(D \frac{\partial c}{\partial x}\right)
$$

(4) Chloride ion corrosion

$$
C(x, t)=C_{0}+\left(C_{s}-C_{0}\right)\left[1-\operatorname{erf}\left(\frac{x}{2 \sqrt{D t}}\right)\right]
$$

Typically, the chloride ion corrosion to concrete includes chloride ion penetration period (i.e., rusting induction period $\mathrm{t}_{0}$ ) and corrosion expansion period, where corrosion expansion period can be divided into corrosion development stage and corrosion damage stage, as shown in Figure 1.

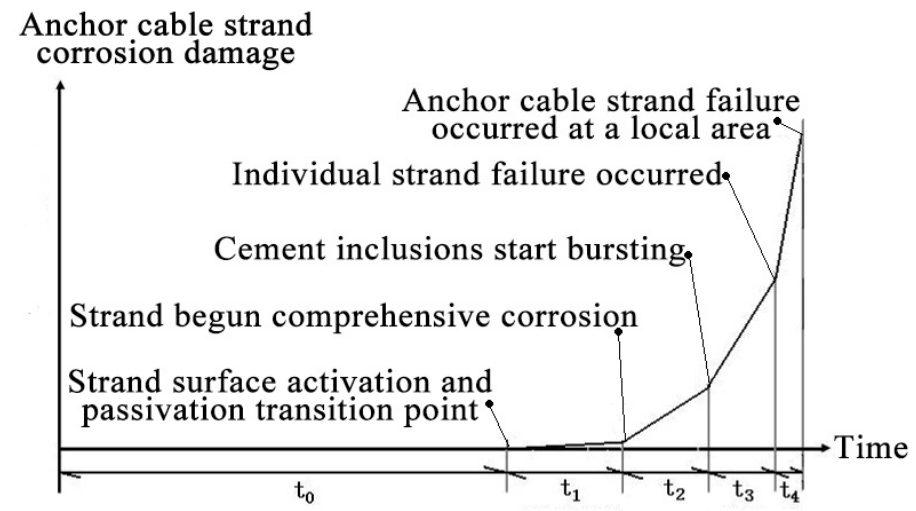

Fig. 1 Concrete corrosion damage process

Studies have shown that the corrosion development period is shorter than the corrosion induction period. Therefore, a lot of studies currently consider that the durable life of concrete ends when chloride ion corrosion occurs to the reinforcement surface and the critical concentration is reached.

Accordingly, the life of anchor structure ends until chlorine ions in the rock and soil invade into the concrete and their concentration arrives at the critical concentration during the study in this paper. 


\section{Prestressed anchor corrosion life prediction model}

In the actual corrosion by chloride ions, the time when chloride ion corrosion occurs to the reinforcement surface should be a random variable with certain probability distribution features due to the randomness of concrete quality and variability of environmental conditions. Use of probability of chloride ion corrosion to concrete structure will be conducive to solving the durability of concrete structure with a more mature reliability theory. Based on these considerations, this paper focuses on discussing the prediction of durable life of anchor cable under chloride ion corrosion based on the specified reliable indicators and the status quo of domestic and foreign studies and analysis of chloride ion corrosion.

\subsection{Anchor structure corrosion}

Structural reliability means the capabilities and properties of a structure to complete predetermined functions within the specified time limit and under the specified conditions. Structural reliability is a measure of reliability, which is defined as the probability for an engineering structure to complete a predetermined function within the specified time limit and under the specified conditions.

If the structure or part thereof cannot meet some requirements of a design as specified by the design after a certain state, this specific state is the limit state corresponding to this function.The function of structure is defined as $Z=g\left(X_{1}, X_{2}, \cdots, X_{n}\right)$, where, $X_{1}, X_{2}, \cdots, X_{n}$ represents the main factors affecting the reliability of an engineering structure. If all basic variables affecting all aspects of the structural resistance are expressed as resistance $\mathrm{R}$ and all basic variables affecting action effect are expressed as action effect $\mathrm{S}$, the function of structure may be

expressed as:

$$
Z=R-S
$$

When $Z>0$, the structure is at the reliable state; $Z<0$, the structure is at the failed state; $Z=0$, the structure is at the ultimate limit state;

If the probability density function of any variable in $\mathrm{R}$ and $\mathrm{S}$ and the probability distribution function of the other variable are known, the expression of failure probability is derived from the probability theory equation:

$$
P_{f}=1-P_{s}=1-\int_{-\infty}^{\infty} f_{s}(s)\left[\int_{s}^{\infty} f_{R}(r) d r\right] d s=\int_{-\infty}^{\infty} F_{R}(s) f_{s}(s) d s
$$

In order to simplify the calculation of reliability index $\beta=\frac{m_{z}}{\sigma_{z}}$, where, $m_{z}$ and $\sigma_{z}$ are the mean and standard deviation of $Z$. $\beta$ and failure probability $P_{f}$ have the following relationship:

$$
P_{f}=\phi\left(-\frac{m_{z}}{\sigma_{z}}\right)=\phi(-\beta)
$$

When the standard deviation $m_{z}$ is constant, the reliability index $\beta$ is only affected by the mean $m_{z}$. When the reliability index increases, the probability density curve will move to the right, which will reduce the shaded area in Figure 2 and the corresponding structural failure probability. 


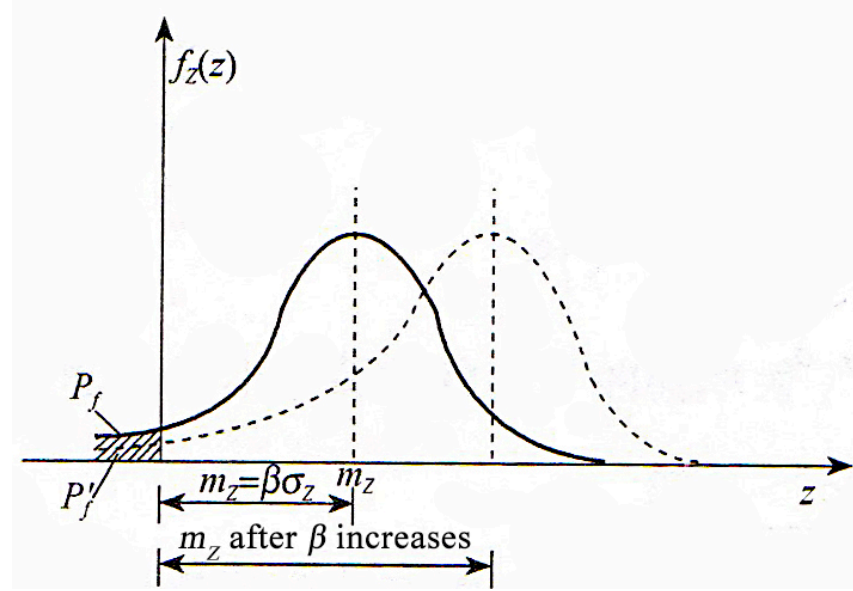

Fig. 2 Physical meaning of reliability index

\subsection{Prediction of durable life of concrete structure based on the specified reliability index}

This paper proposes a durable life prediction method based on the specified reliability index. The basic idea of this method is: Due to chloride ion corrosion, the durability of concrete structure will gradually be reduced, and its durability and reliability will also be gradually weakened. When the reliability is lower than the set reliability index, it is considered the durability of structure arrives at a certain ultimate limit state and its durable life ends.

(1)Limit state of durability due to chloride ion corrosion

In case of poor operation conditions or lower safety performance of a structure due to insufficient durability, the reliability index proposed by Siemes and Rostam may be used ${ }^{[8]}$. For the standards for settings of limit state of durability of chloride ion corrosion, see Table 1.

Table 1 Standards for settings of limit state of durability due to chloride ion corrosion

\begin{tabular}{ccc}
\hline Limit state & Event & Reliability index $\beta$ \\
\hline Serviceability limit state & Corrosion starts & $1.5-1.8$ \\
\hline
\end{tabular}

(2)Failure mode of durability due to chloride ion corrosion

Considering that the key point of failure of structural durability induced by chloride ion corrosion is that reinforcement corrosion starts, this paper uses it as the main mode for failure of durability. When the chloride ion corrosion depth is equivalent to the minimum thickness of cover, it is considered that the failure of durability occurs.Based on the above considerations, the function of reliability of durability due to chloride ion corrosion $Z$ may be defined as the difference between structural resistance $R$ (cover thickness) and applied load $S$ (time-varying chloride ion corrosion depth).

(3)Calculation of durable life due to chloride ion corrosion

For chloride ion corrosion, it is considered based our model that the cover thickness is a function of position, will not change over time, and thus defined as $R=f(x)$. Fick's second law shows that chloride ion corrosion depth $\mathrm{S}$ should be the function of time $\mathrm{t}$ and spatial position $\mathrm{x}$, which satisfies $S=2 \operatorname{erf}^{-1}\left(\frac{C_{S}-C_{c r}}{C_{S}-C_{0}}\right) \sqrt{D t}$ then the equation of limit state of structural durability in the environment of chloride ion corrosion is:

$$
Z=R-S=f(x)-2 e r f^{-1}\left(\frac{C_{s}-C_{c r}}{C_{s}-C_{0}}\right) \sqrt{D t}
$$

Then the failure probability is:

$$
\left.P_{f}=P\left(f(x)-2 e r f^{-1}\left(\frac{C_{s}-C_{c r}}{C_{s}-C_{0}}\right) \sqrt{D t}\right)<0\right)
$$

Where, $\mathrm{x}$ - Distance from concrete surface;

$C_{c r}$ Chlorine ion concentration at time $\mathrm{x}$;

$C_{\mathrm{s}}$ - Chloride ion concentration at the exposed surface of concrete; 
$C_{0}$ - Initial chloride ion concentration in the concrete;

$D$ - Diffusion coefficient of chlorine ions in concrete.

From a security point of view, at the design stage, the calculated reliability index $\beta$ should be greater than the minimum acceptable level of reliability set based on the security level of structure. In accordance with the standards for settings of durability limit state and some of the findings, this paper will take 1.5 for $\beta$ as the specified minimum reliability index of durability limit.

\subsection{Estimation of probability density based on support vector machine (SVM)}

SVM probability density estimation method ${ }^{[9,10]}$ is a non-parametric probability density estimation method and will produce good estimation results of small sample data such as thickness of concrete cover and diffusion coefficient of chlorine ion; therefore, this paper mainly uses SVM for probability density estimation.

The kernel function in the image space is defined as:

$$
K\left(x_{i}, x_{j}\right)=\sum_{r=0}^{\infty} \psi_{r}\left(x_{i}\right) \psi_{r}\left(x_{j}\right)
$$

Its corresponding cross kernel function is:

$$
\kappa\left(x_{i}, t\right)=\sum_{r=0}^{\infty} \psi_{r}\left(x_{i}\right) \phi_{r}(t)
$$

Therefore, after obtaining the solution of linear regression function $w$, the solution of linear operator equation is derived from the cross kernel function:

$$
f(t)=\sum_{i=1}^{l}\left(\alpha_{i}^{*}-\alpha_{i}\right) \kappa\left(x_{i}, t\right)
$$

\section{Life prediction of reinforced concrete due to chloride ion corrosion based on Qinshan Nuclear Power Plant}

Qinshan Nuclear Power Plant is located in the north of Hangzhou Bay, whose water is characterized by low salt due to inflow of the Qiantang River, rainwater and seawater outside the Bay and which is located at the fresh seawater basin.This paper uses the materials and data collected at Qinshan Nuclear Power Plant and obtains the chloride ion concentrations of anchor cable at different heights and different positions after rigorous treatment of samples so that the model is constructed based on the reliability theory of practical problems, and further validate the applicability of methods in this paper.

\subsection{Description of experimental data}

(1) Reinforced concrete cover thickness

In order to more accurately reflect the thickness of cover to reinforcement in concrete, core samples were respectively taken from 9 positions after the 12.5th year (drill 2) and the 13.5th year (drill 1), this paper selected randomly 32 sample points for probability density estimation of cover thickness $f(x)$. Using SVM regression analysis, the reinforced concrete cover thickness probability density distribution is shown in Figure 3:

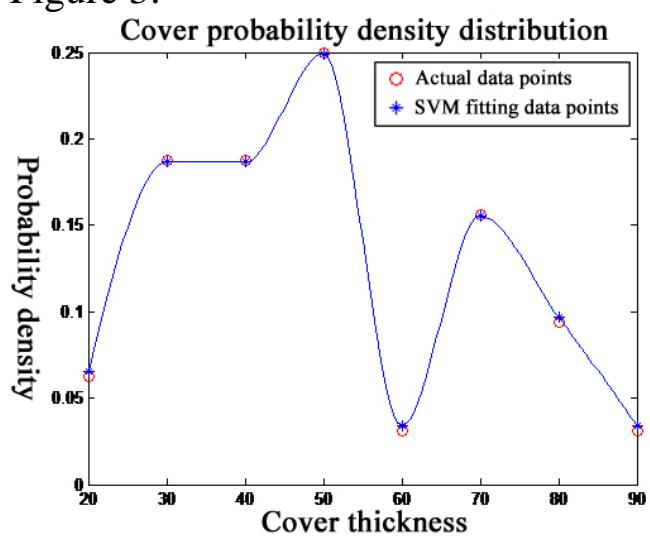

Fig. 3 Cover thickness probability density distribution 
The above figure shows that the use of SVM probability density estimation produces very good regression analysis results, and the probability distributions of cover at $30-50 \mathrm{~mm}$ and $65-75 \mathrm{~mm}$ are relatively large.

(2) Chloride ion diffusion coefficient

Use slices of concrete core samples from the project to determine chloride ion concentration, and derive reversely 27 chloride ion diffusion coefficient values by Fick's second law. Use SVM for regression analysis to obtain the distributions of chlorine ion probability density at the 13.5th year and the 12.5th year as shown in Figures 4 and 5:

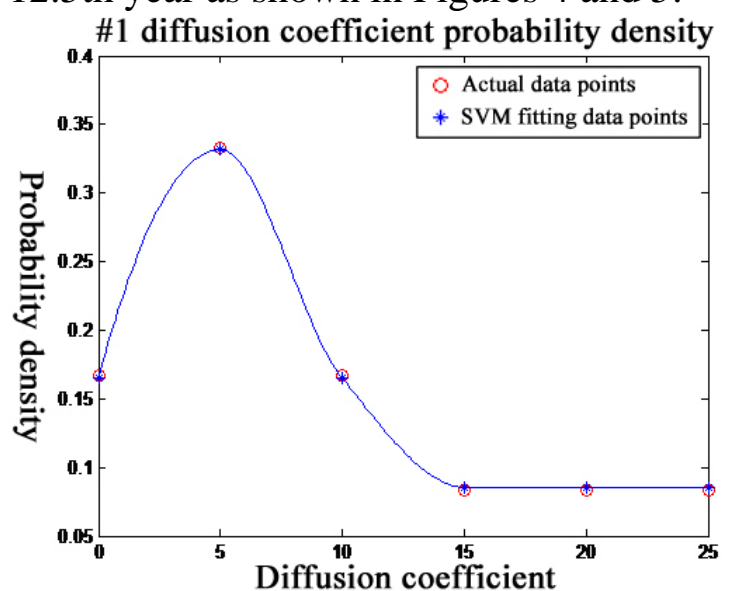

Fig. 4 Chlorine ion probability density distribution in the 13.5th year

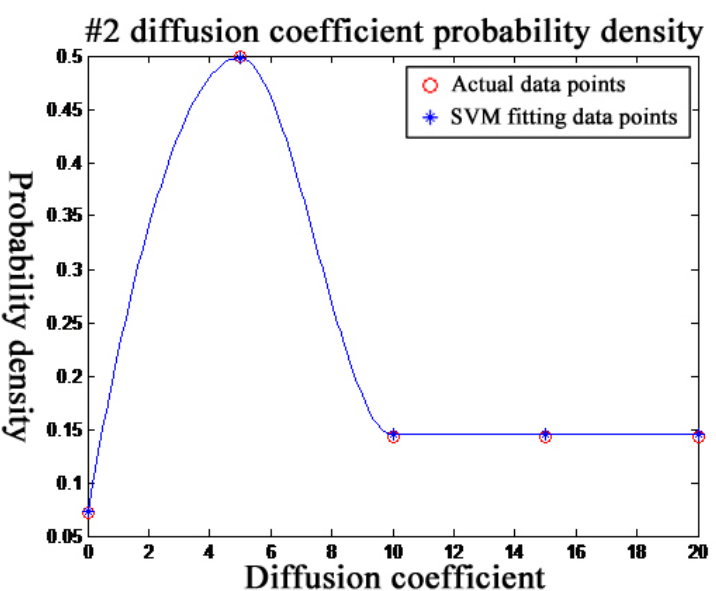

Fig. 5 Chlorine ion probability density distribution in the 12.5th year

Concentration of chloride ions on the surface, critical concentration of chloride ions, and initial concentration of chloride ions are obtained during the reverse derivation by use of Fick's second law.

\subsection{Corrosion life prediction based on reliability theory}

Use the cover thickness and chloride concentration probability density distribution equation in Section 4.1 as well as Equations (6) and (7) and call quad function in MATLAB for definite integral solution to obtain the corresponding failure probability $P_{f}$ of $6.58 \%$ in the 38.15 th year, close to the set probability limit of $6.68 \%$. Thus, we predict that the life of anchor rod arrives at its limit in the 38th year.

\subsection{K-S test method to verify the accuracy of the model}

In order to test the reasonableness of sample probability density obtained by use of SVM method, this paper selects K-S test method under the small sample conditions to test the obtained probability density. If the establishment conditions of K-S test are met, the sample is considered to be consistent with this distribution.

During the K-S test, the most important parameter is $D_{n, a}$, which means the rejected critical value $\mathrm{D}$ when the significant level is $\alpha$ and sample size is $\mathrm{n}$, where $\alpha=0.05$.

After using SVM estimation method to obtain the probability density features of cover thickness, this paper uses K-S test method to further validate its distribution characteristics. Take significant level $\alpha=0.05$, list and calculate observed values of statistic $D$, to obtain the maximum difference $D_{\max }$ of 0.125 , and search the table to get the current rejected critical value $D_{n, a}$ is $0.243, D_{\max }$ is far less than the rejected critical value, and there is no reason to refuse that the thickness of cover is consistent with the SVM estimated distribution.

Use K-S test method to test the characteristics of estimated chlorine ion diffusion coefficient distribution to obtain the maximum difference $D_{\max }$ of 0.098 , and search the table for the current rejected critical value. $D_{n, a}$ is $0.241, D_{\max }$ is far less than the rejected critical value, and there is no reason to refuse that the rejected chlorine ion diffusion coefficient is consistent with the SVM estimated distribution. 


\section{Conclusion}

This paper analyzes the corrosion mechanism of anchor cable in the slope anchor structure, determines that its corrosion is mainly caused by chloride ion corrosion in the ambient environment, selects the more mature reliability theory model in the field of life prediction field based on the characteristics of chloride ion corrosion to analyze its reliability, and uses reliability theory for life prediction.

Since the most critical element in the reliability theory model is the probability distribution type of model parameter, it is necessary to collect a large number of its associated sample to obtain the accurate parameter probability distribution; however, it is very difficult to collect samples from a practical project, it is difficult to obtain a large amount of sample data. This paper uses SVM method to estimate probability density of a small number of samples collected and obtain its probability distribution, the accuracy is beyond the conventional hypothesis test estimation method, and the estimation of small samples is solved. Meanwhile, the application of K-S hypothesis test method further verifies the feasibility of model, which has a good prospect of engineering application and has good practical significance.

\section{References}

[1]. Hong Dinghai. Corrosion and protection of reinforcement in concrete [M]. Beijing: China Railway Publishing House, 1998.

[2]. Cheng Liangkui, Fan Jinglun, Han Jun, and Xu Jianping. Geotechnical anchorage [M]. Beijing: China Building Industry Press, 2003.

[3]. Cai Qilong, Zhu Zhongrong. Corrosion and protection of unbonded prestressed anchor cable [J]. Yangtze River, 2004, 35 (10): 49-51.

[4]. FIP. Corrosion and Corrosion Protection of Prestressed Grout Anchorage, 1986.

[5]. Jin Weiliang and Zhao Xiyu. Durability of concrete structures [M]. Beijing: Science Press, 2002.

[6]. Zhu Anmin. Experimental study of reinforced concrete carbonation and reinforcement corrosion [J]. Shandong Academy of Building Research, 1989.

[7]. Collepardi M, Marcialis A, Turrizzani R. The kinetics of penetration of chloride ion into the concrete[J]. Cem and Concr Res, 1970(4): 157 164.

[8]. Siemes A J, Rostam S. Durable Safety and Serviceability-A Performance Based Design Format[C]. Delft: IABSE, 1996.

[9]. Bean S.J, Tsokos C.P. Development in nonparametric density estimation[J]. Internat Statist Rev, 1990(48): 267-287.

[10]. Chen Xiru, Fang Zhaoben, Li Guoying, et al. Nonparametric statistics [M]. Shanghai: Shanghai Science and Technology Press, 1996.

[11]. Li Fumin, Deng Tianci, Wang Jianghao, and Luo Xiaoya. Review of study on durability of prestressed concrete structures [J]. Journal of Architecture and Civil Engineering, 2015 (32), 1-20.

[12]. Liu Zhenguo. Corrosion behavior of prestressed anchor structures [D]. Beijing: China University of Mining and Technology, 2014.

[13]. Zhao Fang. Marine concrete structure durability design and life prediction parameter analysis based on reliability [D] Shandong: Yantai University, 2013.

[14]. Sun Congtao. Study on concrete durability and life prediction based on chloride ion corrosion [D]. Shanxi: Xi'an University of Architecture and Technology, 2010. 
[15]. Fu Yandi. Life prediction and sensitivity analysis of concrete structures under chlorine erosion [D]. Qingdao:Qingdao Technological University,2010. 